\title{
Estado y frontera en el norte de Chile ${ }^{1}$
}

\section{State and border in Northern of Chile}

Viviana García Pinzón*

Resumen

A propósito del fallo de la Corte Internacional de Justicia en el diferendo por los límites marítimos entre Perú y Chile (enero de 2014) y de la demanda interpuesta contra este último país por parte de Bolivia, la frontera norte de Chile ha ganado renovada visibilidad en los medios de comunicación y en la agenda política chilena. Más allá de dicha coyuntura, las dinámicas transfronterizas y de integración regional y la consolidación de una nueva agenda de seguridad han llevado al posicionamiento estratégico de este territorio, históricamente aislado y marginado en medio del excesivo centralismo que ha caracterizado a la institucionalidad estatal chilena. A lo largo del artículo se analizan los factores que dan cuenta de la importancia de esta zona en el ámbito regional e internacional, así como la agenda en términos de política pública para este territorio por parte del Estado chileno.

Palabras clave: frontera, norte de Chile, política pública, globalización, territorio.
Abstract

As a result of the ruling of the International Court of Justice in the dispute over the maritime boundary between Peru and Chile and the lawsuit filed against the latter by Bolivia, Chile's northern border has gained renewed visibility in both the media and the Chilean political agenda. Beyond this situation, the cross-border and regional integration dynamics and the consolidation of a new security agenda have led to the strategic positioning of this territory, historically marginalized and isolated in the midst of excessive centralization that has characterized the Chile's state institutions. In that sense, the factors that account for the importance of this area in the regional and international levels, together with the agenda in terms of the Chilean government's public policy for this territory, are analyzed throughout the article.

Keywords: boundary, northem Chile, public policy, globalization, territory.

Recibido: 16 de febrero de 2014.

Aprobado: 10 de noviembre de 2014.

${ }^{1}$ El artículo es resultado del proyecto de investigación "Entre el conflicto y el aislamiento: Desarrollo, gobernabilidad y seguridad en las zonas fronterizas del norte y el sur de Chile a comienzos del siglo xxi" (Fondecyt № 1120405-Chile).

* Universidad de la Salle, Facultad de Ciencias Económicas y Sociales. Dirección: Calle 25d, No. 36-07, Bogotá, Colombia. Correo electrónico: vivianagarciapinzon@gmail. com 


\section{Introducción}

Desde la década de 1990 la discusión sobre el concepto de frontera y su pertinencia ha renovado su importancia en las ciencias sociales (Andreas, 2003; Newman, 2003 y 2006; Tapia, 2012; Villafuerte, 2009). Al respecto, Tapia (2012) señala que la discusión sobre las fronteras dentro de la teoría de la globalización puede clasificarse en dos cuerpos de interpretación: por una parte, aquellos que consideran que la globalización es un fenómeno que perfora las fronteras y debilita la función separadora que les fue atribuida tradicionalmente. Por otro lado, hay una corriente que sostiene que la globalización no supone necesariamente el debilitamiento de las fronteras, "[...] lo que se observa es un fenómeno de fronterización, es decir de cierre de fronteras y endurecimiento de los requisitos para la circulación de las personas" (Tapia, 2012, p. 179). Con base en esta perspectiva, la globalización ha llevado a un redescubrimiento de las fronteras, donde lo periférico ha cobrado centralidad. "No se trata de espacios accesorios, sino de ámbitos estratégicos que ejercen nuevas funciones, separar lo bueno de lo malo. La frontera marca la diferencia entre lo incluido y lo rechazado" (Villafuerte, 2009, p. 694).

En este sentido, Peter Andreas (2003) señala que dentro de la teoría de la globalización se habla del declive de las fronteras, a partir de una visión de éstas como puentes para transacciones comerciales más que como barreras económicas o líneas militares fortificadas. Sin embargo, si bien la globalización puede haber socavado los límites económicos, ha traído consigo también nuevas funciones y énfasis respecto del control del Estado en los límites territoriales. Paradójicamente, mientras la globalización ha estimulado la libre circulación de capitales y bienes - cuestionando la soberanía estatal en este ámbito-, las formas de control territorial por parte del Estado se mantienen y se han enfocado en impedir el flujo de ciertos bienes, considerados ilícitos, y también de personas, todo lo cual es considerado como una problemática de seguridad.

La persistencia de disputas geográficas entre Estados, la continuidad y emergencia de nuevos conflictos por el territorio, así como los esfuerzos de los Estados por controlar las zonas limítrofes frente a las nuevas amenazas trasnacionales, evidencian la importancia del territorio, del espacio donde tiene lugar la interrelación entre fenómenos globales y realidades y 
prácticas locales. Las zonas fronterizas son un espacio particular que ofrece una mirada a la naturaleza de las políticas territoriales y las dinámicas de seguridad en el siglo xxi.

En un artículo publicado en Foreign Affairs Latinoamérica, Claudio Fuentes (2008) usaba el adjetivo de "calientes", como sinónimo de "conflictivas”, para referirse a las fronteras en América Latina y el Caribe. Según este autor, debido al bajo número de guerras interestatales y guerras civiles vigentes, la región es considerada como una de las regiones más pacíficas del mundo. Sin embargo, los conflictos territoriales irresueltos entre los Estados, la desconfianza en las relaciones estatales, el crecimiento de las amenazas trasnacionales, y la debilidad de los Estados configuran un escenario donde los límites fronterizos han adquirido gran importancia como espacio de potenciación de las problemáticas de seguridad, así como de desarrollo de iniciativas de cooperación que, a su vez, estimulan la integración.

A partir de las anteriores consideraciones, este artículo desarrolla un análisis sobre los factores de inserción internacional y regional (Sur América) de la frontera norte de Chile, ${ }^{2}$ y las funciones y forma de territorialización del Estado chileno. Algunos de las interrogantes que guían la reflexión son: ¿Cuál es la importancia del territorio de la frontera norte en el contexto regional e internacional desde la visión del Estado chileno?, ¿̇enómenos como la globalización y la integración regional han traído consigo cambios en el lugar de las fronteras en la política pública y la política exterior en Chile?, y ¿qué lugar ocupa la dimensión territorial en el accionar estatal respecto de las fronteras?

La emergencia de nuevos actores no estatales y de procesos transfronterizos impulsados por los gobiernos subnacionales o por las comunidades, ha motivado el desarrollo de diversos trabajos y reflexiones en torno a la frontera norte chilena. En este sentido, hay un buen conjunto de análisis sobre las dinámicas transfronterizas y paradiplomacia, la mayoría enfocados al estudio de la Alianza Estratégica "Aymaras sin fronteras" (Aranda, Ovando y Corder, 2010; Bello, 2012; González, 2009; González y Ovando, 2008; González, Rouviere y Ovando, 2008; Ovando y Álvarez, 2011). A

${ }^{2}$ La frontera norte chilena limita con tres países: Bolivia, Perú y Argentina, y comprende una parte del territorio de las regiones de Arica y Parinacota XV, Tarapacá I, Antofagasta II. 
éstos se suman, las investigaciones en torno a fenómenos sociales propios de la frontera que se han visibilizado en las décadas recientes, tales como la inmigración desde Bolivia y Perú (Cano y Sofía, 2009; Tapia, 2012). Finalmente, hay una abundante producción sobre la frontera que se inscribe en una perspectiva más tradicional enfocada en las relaciones vecinales en el marco de las tensiones territoriales y la política exterior chilena. No obstante, la revisión de la literatura sobre el tema denota un menor desarrollo en la reflexión sobre los cambios en la agenda del Estado chileno en el contexto de la globalización y el efecto que ello ha tenido en el modelo de territorialización y las funciones en las áreas fronterizas.

Frente a esto, los objetivos de este trabajo son dos: por un lado, caracterizar la frontera norte de Chile y analizar los factores de su inserción regional e internacional en relación con la institucionalidad estatal. Por otra parte, mediante el estudio de caso, se busca aportar a las interpretaciones existentes respecto de la frontera y la globalización a partir de evidencia empírica que contribuya a esta discusión. Para ello, se estructura un marco teórico que recoge aportes de diversas disciplinas señalando los cambios en las formas de territorialización y función del Estado en las fronteras dadas las transformaciones del capitalismo; asimismo, se realizó una revisión de documentos oficiales y bibliografía especializada que permitió la identificación de las trayectorias y los puntos de cambio en la relación entre la institucionalidad estatal y el territorio fronterizo del norte de Chile.

En el texto se sostiene que, desde una perspectiva top-down, el área de frontera del norte de Chile constituye a la vez una frontera interna e internacional. En su condición de frontera interna, se caracteriza por su marginalidad y exclusión histórica respecto de los centros de poder nacional. ${ }^{3}$ Sin embargo, como frontera internacional, las dinámicas económicas y sociales en el marco de la globalización han generado una inusitada importancia de esta zona en torno a tres variables: comercio y exportación de bienes primarios, infraestructura y seguridad.

3 "Cabe señalar que el Estado chileno se ha referido a estos territorios bajo el concepto de frontera interior, entendida como aquella sobre la cual al Estado chileno le 'cuesta alcanzar institucionalmente'. Loreto Correa Vera, entrevista, 11 de septiembre de 2014. Profesora Titular, Academia Nacional de Estudios Políticos y Estratégicos, Santiago, Chile. 
El artículo está organizado de la siguiente manera: en la primera parte, se presenta una revisión teórica sobre límites, fronteras y territorio. En la segunda, hay una caracterización general del territorio que comprende la frontera norte de Chile. En el tercer acápite se abordan y analizan las variables que dan cuenta de la importancia e inserción regional e internacional de esta zona. Finalmente, se presentan algunas conclusiones con base en el análisis precedente.

\section{Territorio, Estado y fronteras}

La noción de frontera ha sido utilizada para denominar realidades fácticas, metafóricas y/o imaginarias construidas social o disciplinariamente; se trata de un concepto polisémico al que se recurre con distintas acepciones dentro de las ciencias sociales (Bartolomé, 2005; Tapia, 2012). No obstante, la mirada tradicional sobre las fronteras estatales remite a la paz de Westfalia (1648), a partir de la cual se sentó una visión que concibe a las fronteras como demarcaciones de los Estados nacionales y de las sociedades que los habitan (Tapia, 2012, p. 179). Al respecto, Gottman (1973, citado por Machado, Reyes y Rego, 2009, p. 99) señala que en geografía política una frontera internacional es considerada como una zona de indeterminada anchura cuya característica fundamental es que está cruzada por una línea de límite que determina los límites de la soberanía territorial entre dos Estados. Siguiendo esta definición clásica, se encuentra que el estudio de las fronteras ha estado asociado a las líneas que separan la soberanía de los Estados en el sistema internacional (Newman, 2003); ésta es una visión geopolítica de la frontera como elemento de demarcación de la soberanía de un Estado, que ha llevado a que en gran parte de la literatura limite territorial y frontera sean tratados como sinónimos.

Sin embargo, hay una diferencia entre los límites fronterizos y las áreas de frontera: el límite se concibe como la constitución de líneas de separación físicas y visibles entre espacios económicos, políticos y sociales. Entre tanto, el área de frontera es la región o área en relativa proximidad al límite, donde las dinámicas de cambio y las prácticas cotidianas están afectadas por la misma presencia del límite (Newman, 2006). Como bien lo señala Lía Machado (2000), mientras los límites fronterizos son obra de los 
Estados que a través de instrumentos jurídicos marcan la separación entre unidades políticas soberanas y delimitan el territorio bajo control estatal, las áreas de frontera son construcciones sociales de los pueblos, los cuales se pueden expandir más allá del límite jurídico del Estado, desafiar la ley territorial de cada Estado limítrofe y a veces crear una situación de facto potencialmente conflictiva.

Las dinámicas y fronteras culturales, económicas y sociales no siempre coinciden con los límites estatales; de manera tal que las áreas de frontera son espacios de movimiento, intercambio e integración en variadas formas, cuyo desarrollo territorial se da en un marco que va más allá de la división político-administrativa de los Estados, integrando actores subnacionales y actores no estatales. Así, las fronteras son una construcción socio-territorial producto de los discursos jurídicos y geopolíticos de los Estados, por una parte, y de la acción de las comunidades fronterizas, por otra.

Tradicionalmente, el estudio de las fronteras se ha enfocado en la mirada sobre los límites fronterizos, es decir, una visión de "arriba hacia abajo" (top-down) que ha privilegiado el accionar estatal en el análisis. Desde las relaciones internacionales las fronteras han sido abordadas en términos militares y geopolíticos, principalmente. Dado que la mayoría de las guerras interestatales han sido por la conquista del territorio, el pensamiento geopolítico ha enfatizado la centralidad de la competencia y la adquisición del territorio en las dinámicas de la política mundial y la función militar de las fronteras. En este sentido, la sobrevivencia del Estado depende del combate contra las incursiones militares de otros Estados, perspectiva que coincide con la tradición teórica realista en la política internacional, en la cual la seguridad del Estado está estrechamente vinculada con la guerra. Pero el declive de las guerras interestatales y las transformaciones en la política mundial han demostrado la debilidad de este enfoque para la compresión de las relaciones entre actores en el sistema internacional.

Así, en lo que se refiere al estudio de las fronteras poco a poco se ha posicionado una mirada desde "abajo hacia arriba" (bottom-up), la cual aborda las experiencias de los individuos y las poblaciones y las formas en que las fronteras repercuten en las prácticas cotidianas y las identidades de las personas que las habitan (Grimson, 2000; Tapia, 2012). Esta es una mirada que ha sido impulsada principalmente por la antropología y la sociología desde finales de la década de 1970 (Grimson, 2000, 2003 y 
2004; Wilson y Donnan, 1998), y que supone una crítica al nacionalismo metodológico y privilegia una visión constructivista y multicultural en sus discursos, deconstruyendo las visiones geopolíticas y militares características de los discursos de Estados nacionales sobre las fronteras.

Pero ese esfuerzo por deconstruir las identificaciones nacionales se ha dado a costa de un excesivo énfasis en reconocer la "inexistencia" de las fronteras para los actores locales, siendo que la administración de las fronteras está imbricada en la definición contemporánea del Estado-nación y, a pesar de la pérdida de soberanía estatal en campos tales como la economía, el Estado sigue reclamando una posición central en el ordenamiento de flujos dentro y fuera de los límites nacionales. Harvey (1990) señala que los conceptos de tiempo y espacio son una construcción social vinculada de manera estrecha con las formas de producción y las formas de reproducción social. De manera que las dinámicas actuales del capitalismo han llevado a un replanteamiento en el modelo de territorialización del Estado y sus funciones en las áreas de frontera.

$\mathrm{Al}$ analizar las dinámicas de control estatal en zonas fronterizas de América Latina, Grimson (2004) anota que:

[E]l control sobre las poblaciones fronterizas parece haberse fortalecido, tanto en relación a la circulación de personas como de pequeñas mercaderías del llamado "contrabando hormiga". Así, en muchos casos, los pobladores fronterizos perciben una mayor - no una menor- presencia estatal. El estado se retira en su función de protección y reaparece en su papel de control y regulación. En otras palabras, podríamos estar asistiendo —más que a una desterritorialización generalizada- a la sustitución de un modelo de territorialización por otro (p. 11).

Ese nuevo modelo de territorialización trae consigo una variación histórica en las prioridades de control fronterizo; a medida que las fronteras se hacen cada vez más porosas por efectos de la globalización, los Estados han construido barreras contra el flujo de elementos "indeseables", contra la ilegalidad. Así:

[E]l estado no se ha retirado completamente, sino que ha cambiado su eje de intervención. Si en la fase anterior su obsesión era la preservación territorial, 
el control del espacio, ahora su eje de acción se vincula a controlar los flujos, los movimientos de personas y mercaderías entre los países. Especialmente a promover los "flujos por arriba" y controlar los flujos por "abajo" (Grimson, 2004, p. 12).

Aguiar (2010) habla del "neoliberalismo selectivo" para dar cuenta de esta dinámica; así, mientras el neoliberalismo promueve una ideología de libre mercado y la reducción del control del Estado, también nuevas barreras son construidas. "Las zonas de frontera pasaron de tener un tipo de valor instrumental a otro, en el sentido de que la hegemonía militar y territorial es desplazada con el neoliberalismo por la hegemonía del container y los flujos entre las transnacionales" (Grimson, 2004, p. 18).

\section{La frontera norte de Chile: Entre la integración y el aislamiento}

Muchas de las áreas de frontera en Suramérica pueden ser descritas como "tierras marginales" respecto de los centros de poder nacional desde la independencia del dominio colonial.

Durante el período colonial, tanto España como Portugal invirtieron en el establecimiento de límites para asegurar la división colonial de sus posesiones coloniales en un nivel mayor que los gobiernos de post-independencia. Los nuevos estados independientes mantuvieron el patrón dominante de asentamiento caracterizado por la concentración de la población en la región de la Cordillera de los Andes a lo largo de la costa del Pacífico y del Atlántico en la zona occidente de Sur América. La mayoría de las fronteras internas se mantuvieron marginales a los principales flujos de asentamiento (Machado et al., 2009, p. 100-101).

El hecho de que la formación y consolidación de la institucionalidad del Estado haya seguido una trayectoria de expansión centro-periferia en la mayoría de los Estados suramericanos, ha dejado a las zonas fronterizas en un plano secundario. En efecto, el espacio terrestre bajo soberanía es más amplio que la presencia estatal efectiva; existiendo un desequilibrio entre población, territorio, desarrollo e institucionalidad. Por ello, cuando 
se habla de fronteras en Suramérica se alude a un doble proceso: son a la vez fronteras internas y fronteras internacionales (Ramírez y Cepik, 2004, p. 450$)$.

La frontera norte chilena limita con tres países: Bolivia, Perú y Argentina, y comprende franjas territoriales de las regiones de Arica y Parinacota Xv, Tarapacá I, Antofagasta II —conocidas también como el Norte Grande por la clasificación regional desarrollada por la Corporación de Fomento para la Producción (CORFo) en 1950—. Si bien no se debe confundir la frontera norte, como un espacio territorial específico, con el Norte Grande de Chile, la caracterización de este último es importante para comprender el contexto geográfico, político, económico y social del área fronteriza. Al respecto, cabe mencionar que se trata de una amplia porción del territorio chileno; con una superficie de $185.148,2 \mathrm{~km}^{2}$, es una región caracterizada por un clima desértico y que concentra tan sólo $6.3 \%$ de la población total de Chile (INE, 2012), siendo Arica y Parinacota la región menos poblada (1.29\%).

La institucionalidad del Estado chileno, marcada por una trayectoria de expansión centro-periferia y el predominio de una cultura centralista, ha implicado, entre otras cosas, la circunscripción modelo de desarrollo y el accionar estatal al Valle Central, dejando a las regiones fronterizas del norte y sur del país en un plano secundario. Pero este hecho no es propio o exclusivo del Estado chileno. De hecho, los territorios de los cuatro países que convergen en la frontera se encuentran alejados de los centros económicos y político-administrativos nacionales, de manera que éstas constituyen a la vez fronteras internas y fronteras internacionales.

Desde el año 1960, la preocupación por el acondicionamiento del territorio y los problemas derivados de la excesiva concentración geográfica de hombres y medios de producción en la capital se hace sistemática en Chile (Szary, 1997). Bajo este contexto, en 1974 se llevó a cabo un proceso de regionalización en Chile, a partir del cual las regiones adquirieron el estatus de entidades administrativas. Navarrete e Higueras (2014) señalan que este modelo se dio "en un marco de políticas neoliberales, donde los motivos de su desarrollo se vinculaban con razones políticoestratégicas de integridad e inclusión nacional y la prestación de bienes y servicios de manera eficiente y eficaz a la ciudadanía” (p. 184). Desde entonces, y particularmente luego de la reinstauración democrática en 1990, 
se han implementando algunas medidas para llevar la democracia a los niveles regionales y locales; éstas se han concentrado en procesos de descentralización administrativa y fiscal, mientras que las reformas en materia de descentralización política han sido modestas. Sin embargo, más que descentralización, se ha tratado de un proceso de desconcentración, de manera que el mayor peso en las decisiones, planificación y presupuesto permanece en manos del nivel central.

La preocupación por el desarrollo desigual del territorio también ha sido constante en términos de seguridad, bajo la concepción clásica de la soberanía estatal. De hecho, desde el discurso del ejército chileno se posicionó el concepto de fronteras interiores para designar:

[...] espacios terrestres bajo soberanía no vinculados total o efectivamente a la acción del gobierno central y/o regional donde se dificulta el desarrollo de las actividades humanas y productivas por su distancia del núcleo vital geohistórico del país; la ausencia de vías de comunicación, influencia económica y cultural extranjera y la percepción ciudadana colectiva en cada uno de los espacios geográficos de construir un ente diferente, separado, postergado del resto de la población nacional, y, que no alcanza a disfrutar del bienestar general debido a factores geográficos adversos (Garay, 2004, pp. 99-100).

En el año 1994, el presidente Eduardo Frei afirmaba: "Mi gobierno está decidido a hacer una plena ocupación de la tierra, mar y aire de nuestra patria. Consolidaremos e integraremos las fronteras interiores y haremos una ocupación efectiva de nuestra tierra" (Garay, 2004, p. 100). En ese mismo año fue creado el Comité de Fronteras Interiores, el cual estaba integrado por el ministro de Defensa, los subsecretarios de Guerra, de Desarrollo Regional, de Obras Públicas y de Bienes Nacionales, el director de Fronteras y Límites, el comandante del Comando de Ingenieros del Ejército, el director de la Academia de Guerra de la Institución, el director del Instituto Geográfico Militar y el director del Territorio Marítimo (Martínez, 2014).

En marzo de 1995, la comisión entregó su informe, en el cual identificó a 31 zonas del país como fronteras interiores, lo que equivale a $46.32 \%$ del territorio nacional. Las zonas identificadas están clasificadas a partir de tres categorías: frontera interior no crítica, frontera interior 
intermedia, y frontera interior crítica, "correspondiendo esta última, a aquellas áreas del territorio nacional cuyas características geográficas, de clima, de accesibilidad, de infraestructura y de servicios hacen difícil el desarrollo y la integración de las localidades" (Comisión Nacional sobre Fronteras Interiores al Desarrollo Nacional, 1995, p. 12). En la zona norte del país se identificaron como fronteras interiores críticas: Comunas de General Lagos, Putre, Pica y Colchane en la I Región, esta última fue identificada como crítica, y las comunas de Ollagüe y San Pedro de Atacama en la iı Región.

En el Libro Blanco de la Defensa (Ministerio de la Defensa de Chile, 2010), las fronteras interiores son consideradas como uno de los principales desafíos geográficos.

[L]a existencia de zonas de bajo poblamiento pero de alta potencialidad económica, principalmente en los extremos de nuestro territorio, constituye un factor de debilidad que es necesario superar. En este sentido, el Estado chileno se ha preocupado de implementar medidas tendientes a favorecer a las zonas extremas, de modo de incentivar su desarrollo, poblamiento e integración con el resto del país. Por ejemplo, los planes de desarrollo elaborados para la norteña ciudad de Arica y para la Zona Austral, así como lo relativo a la política de canalización de flujos internos de población hacia esas áreas. Cabe mencionar, además, los esfuerzos realizados por la Subsecretaria de Desarrollo Regional del Ministerio del Interior, junto con el Ministerio de Planificación Nacional (mideplan) y las instituciones de la Defensa, en el análisis de propuestas específicas en materia de "fronteras interiores" (p. 59).

La respuesta del Estado chileno al desigual desarrollo e integración de las regiones en el contexto nacional se ha materializado en un conjunto de políticas públicas enfocadas en lo que se ha denominado "zonas extremas" y "localidades aisladas". ${ }^{4}$

${ }^{4}$ La revisión de la bibliografía al respecto evidencia que han sido utilizados diversos conceptos para identificar a aquellas partes del territorio que se caracterizan por su aislamiento geográfico, baja y dispersa población, y oferta precaria de servicios públicos, entre otros factores; algunos han sido: "zonas de colonización”, "zonas extremas", "fronteras interiores", "territorios aislados" y "territorios especiales". Al respecto, ver BNC, 2011. 
Hasta comienzos de la década de 1990, la cualidad de zona extrema estaba dada principalmente por su lejanía a la zona central del país (ubicación geográfica extrema) y a la condición fronteriza de las áreas en cuestión. Sin embargo, nuevos estudios dieron cuenta de la existencia de territorios que aunque no se ubicaran en zonas geográficas extremas o fronterizas, se encontraban igualmente aisladas — por distintos factores - en relación al resto de las comunas del país (Biblioteca del Congreso Nacional [BCN], 2011, p. 1).

Por ejemplo, en 1994 fue establecido lo que actualmente es el Comité Interministerial para el Desarrollo de las Zonas Extremas y Especiales (CIzEDE), dependiente de la Subsecretaría de Desarrollo Regional y Administrativo, encargado de promover medidas (legislación, programas y proyectos) para la integración de las zonas extremas, aisladas y especiales. Durante la administración del presidente Sebastián Piñera (2010-2014) se estableció una diferencia entre las "zonas extremas" los "territorios aislados", de manera que para los primeros la política ha estado enfocada en el "Plan de Incentivos Especiales para las Zonas Extremas", 6 mientras que en el caso de los "territorios aislados" se ha implementado la "Política Nacional para el Desarrollo de Localidades Aisladas".

La región de Arica y Parinacota es una de las tres regiones consideradas como "zonas extremas". A ello se suma que en el diagnóstico de "localidades aisladas", elaborado en 2008 por la Subsecretaría de Desarrollo Regional y Administrativo, se encontró que tres de las cuatro comunas de Arica y Parinacota se encuentran en el primer cuartil de las más aisladas del país; mientras que de las siete comunas de Tarapacá, tres se encuentran en situación de aislamiento crítico. Para el caso de la región de Antofagasta, dos de las nueve comunas de la región se encuentran en el primer cuartil como las más aisladas del país, y una está en el segundo.

En el ámbito económico, las regiones se han visto expuestas a las fuerzas del mercado con especial ímpetu desde 1973. Siguiendo a Szary (1997):

${ }^{5}$ La región de Arica y Parinacota, en el norte, las regiones de Aysén del General Carlos Ibáñez del Campo y de Magallanes y de la Antártica Chilena, en el sur, y las provincias de Chiloé y Palena conforman las "zonas extremas".

${ }^{6}$ Ver Ley 20.655 de 2013. 
La desregulación permitida por la legislación liberal pone de nuevo el acento, como antes de la crisis de 1929, en las exportaciones con todas las consecuencias que este modelo de crecimiento implica para la infraestructura industrial chilena. Este cuestionamiento de la estructura productiva del país va a conducir a brutales reclasificaciones en los territorios [...] Podemos decir de forma general que el cambio de orientación económica provoca la crisis de las regiones industriales chilenas, en aquellas que frecuentemente habían sido distinguidas como polos de desarrollo (p. 63).

Este modelo económico ha sostenido una tendencia desindustrializante y puso a las regiones a competir con los mercados internacionales, sacando provecho de sus ventajas comparativas, y generando gran dependencia hacia los países inversionistas.

La originalidad de la concepción de las regiones chilenas descansa sin duda en el hecho de que estas han sido pensadas en un doble movimiento de apertura e inserción en el "sistema mundo", antes que en un movimiento de integración territorial nacional [...] Hay una cierta radicalización de las desigualdades regionales, fenómeno que podemos asociar con las diferencias sociales provocadas por el nuevo sistema económico implementado (Szary, 1997, p. 68).

Las cifras del PIB por regiones al año 2012 evidencian la exacerbada desigualdad entre la economía de las regiones y la Región Metropolitana (R. M.) (ver figura 1). Cuarenta y nueve por ciento del PIB regionalizado corresponde a la R. M., le sigue Antofagasta con 10.5\%, el cual está constituido en su mayoría por la explotación minera. Las otras dos regiones que comprenden la frontera norte, Arica y Parinacota y Tarapacá, ocupan un lugar marginal, particularmente Arica y Parinacota cuyo PIB representa $0.63 \%$ del total.

En materia de inversión extranjera, los datos revelan que la gran perdedora en la zona norte del país es la región de Arica y Parinacota ${ }^{7}$ con el

${ }^{7}$ Esta región fue creada en el año 2008, a partir de su separación de Tarapacá. Su creación se debe, "entre otras razones, [a] las especiales condiciones de este territorio y, por lo tanto, en el impacto de éstas en su comunidad, tales como su condición internacional bifronteriza; la distancia a los centros de decisión política tanto regional 


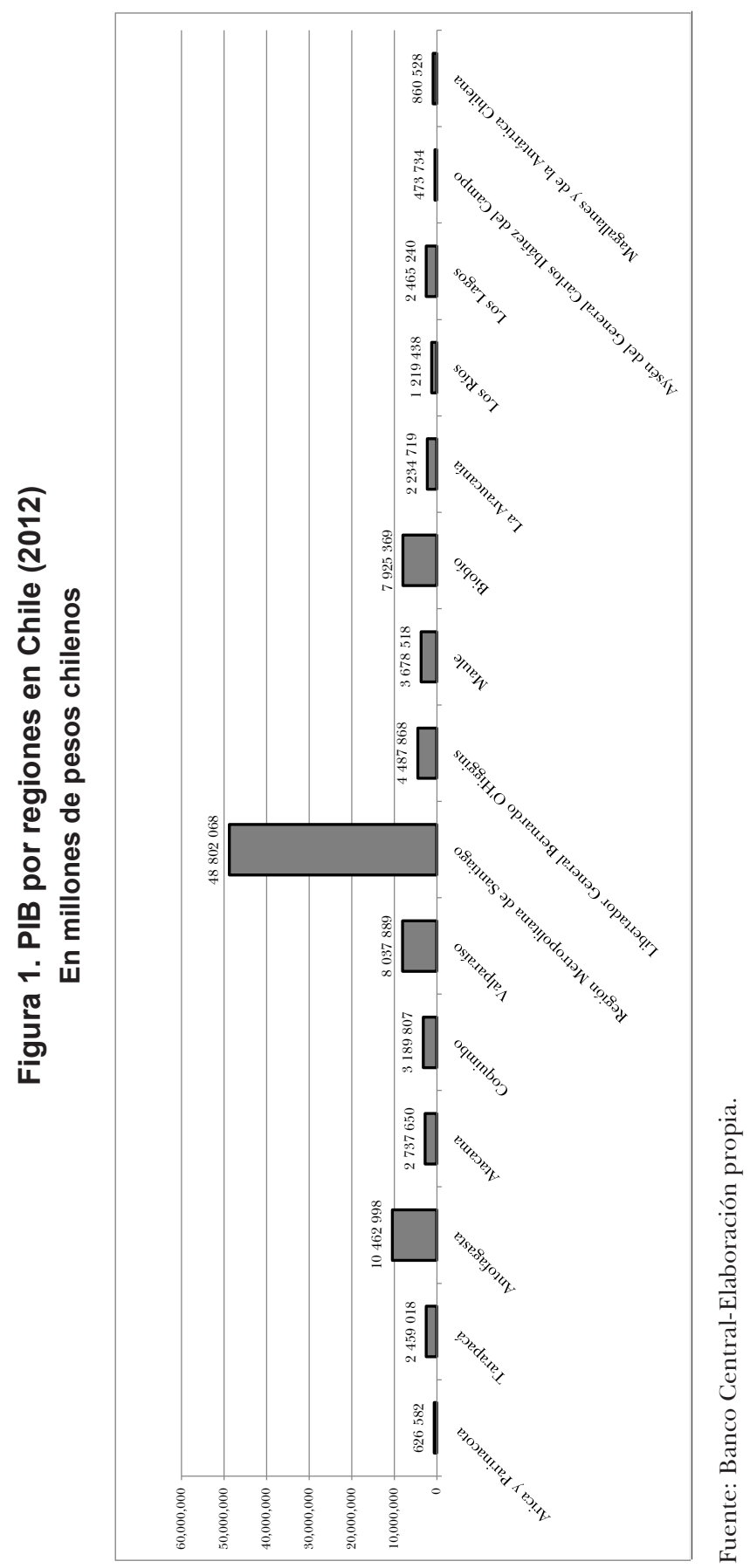


menor porcentaje en el norte del país y la penúltima a nivel general (ver figura 2). Por su parte, la región de Antofagasta es una de las que cuentan con mayor porcentaje, sólo superada por la inversión multirregional y la de la Región Metropolitana. Al analizar los datos por sector, se encuentra que esa alta cifra se debe a la minería, más de $90 \%$ de la inversión en Antofagasta y en Tarapacá está enfocado en este sector, incluso, en el caso de Arica, éste representa casi 50\% (Comité de Inversiones Extranjeras, 2012).

La revisión de la bibliografía sobre el tema y de cifras e indicadores de diversa índole evidencian el aislamiento histórico del territorio del norte del país, no sólo en un sentido geográfico, sino en lo político, social y económico. Esta dinámica de aislamiento envuelve también al área fronteriza del norte, la cual constituye una frontera interna para el Estado chileno.

\section{La frontera norte y su inserción en el contexto regional e internacional}

En su condición de frontera interna, el norte de Chile se ha caracterizado por su marginalidad y exclusión, hecho que ha sido favorecido por el modelo de organización política excesivamente centralizado y por un esquema económico que ha estimulado la inserción de las regiones en los mercados internacionales, en perjuicio de una integración nacional. Sin embargo, como frontera internacional, ese mismo modelo económico y las dinámicas sociales en el marco de la globalización y la integración regional han generado una inusitada importancia de este territorio en torno a tres variables: comercio y exportación de bienes primarios, infraestructura y seguridad.

\section{Relaciones económico-comerciales}

Desde la restauración democrática, en 1990, los ejes articuladores de la estrategia de inserción internacional económica del Estado chileno han sido:

como nacional y contar con una población mayoritariamente de distinto origen étnico cultural, respecto al resto del territorio regional” (BCN, 2007, p. 5). 


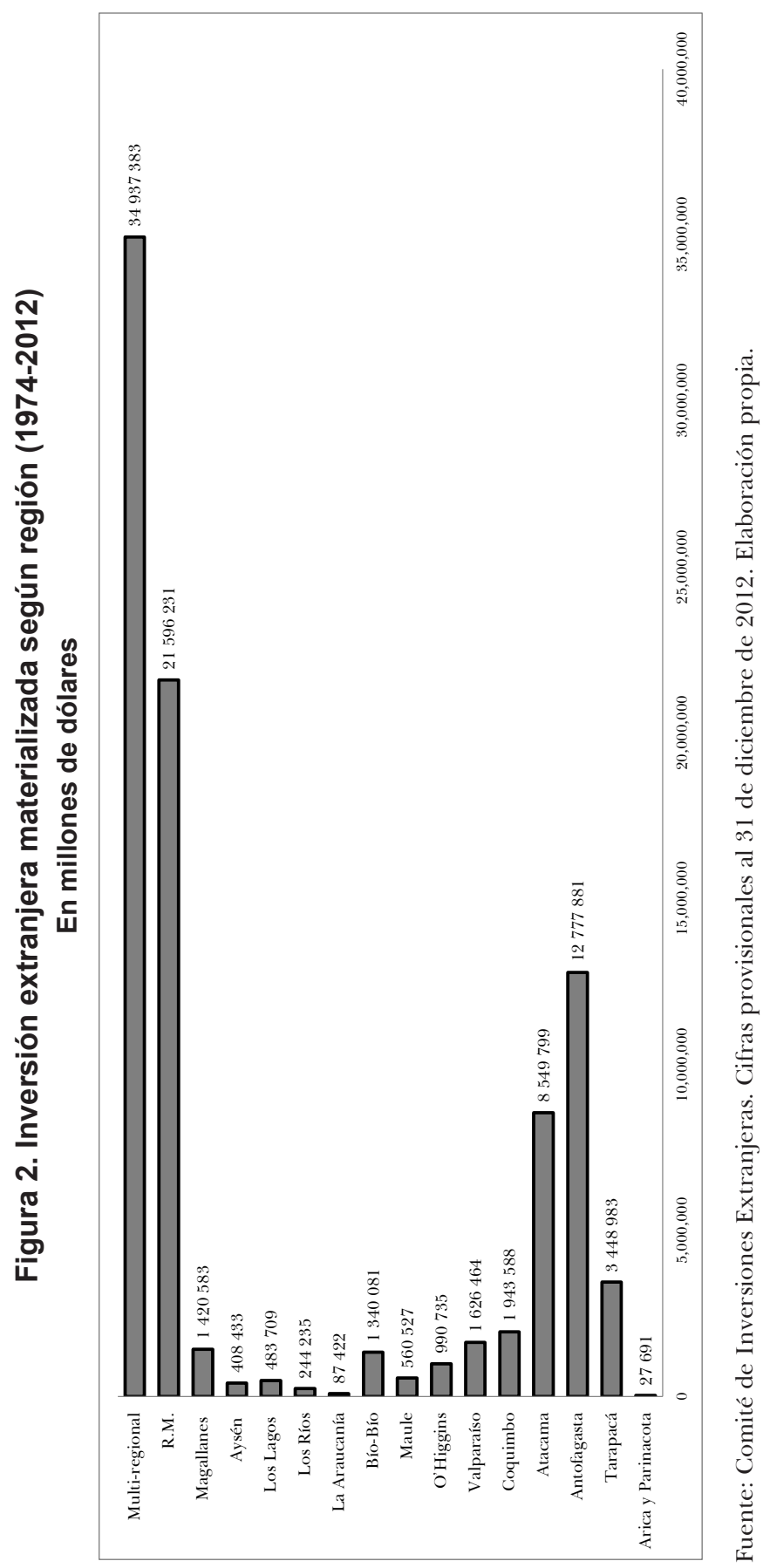


1. La concertación política: El objetivo era la recuperación del prestigio y normalización del papel nacional en los foros internacionales. Ello se tradujo en un dinamismo multilateral y de concertación bilateral en los ámbitos regional y global: "la participación en Naciones Unidas y la candidatura y elección de Chile como Miembro No Permanente del Consejo de Seguridad de Naciones Unidas para el bienio 2003-2004 permitió alcanzar esa meta a nivel global. Paralelamente, la participación en los organismos del sistema interamericano (Cumbres de las Américas y OEA), fueron prueba efectiva del compromiso hemisférico y, por último, el permanente trabajo de la diplomacia nacional en instancias regionales y subregionales como el Grupo de Río, cumbres sudamericanas y el Mercosur, dieron cuenta de las prioridades del gobierno en dicho plano" (Quezada, 2010, p. 126).

2. La integración económico-comercial: Se continuó y profundizó la internacionalización de la economía chilena, enfatizando la promoción de las exportaciones y vinculando el comercio con la inversión extranjera. La apertura unilateral se complementó con un activo bilateralismo que desembocó en tratados de libre comercio y acuerdos de complementación económica (Fuentes, 2011, p. 130).

3. Finalmente, el acercamiento y mejora en las relaciones vecinales: Desde la década de 1990, el gobierno chileno entendió que requería de relaciones cercanas con sus vecinos y no podría proseguir con una estrategia de aislacionismo. El acercamiento vecinal estuvo motivado, en buena medida, por el interés de impulsar iniciativas conjuntas en ámbitos como el intercambio comercial, la integración vial y la cooperación energética (Correa y García, 2012, p. 84).

La frontera norte comprende toda el área límite con Perú y Bolivia y una parte con Argentina. En lo que respecta a Perú, la relación comercial y económica con este país ha cobrado relevancia. Esta relación ha seguido una trayectoria creciente a partir de la entrada en vigencia, en 2009, del Acuerdo de Libre Comercio Chile-Perú. En materia de inversiones, Chile ocupó el sexto lugar en el ranking de Inversión Extranjera Directa (IED) del Perú, representando 6\% de la IED total de este país. "A diciembre de 2013 el stock de inversión chilena directa materializada en ese país alcanzó los 
Cuadro 1. Importaciones y exportaciones con países vecinos (2012) En millones de dólares

\begin{tabular}{|c|lccc|}
\hline \multirow{4}{*}{ Chile } & & Bolivia & Perú & Argentina \\
\cline { 2 - 5 } & Importaciones & 250.300 & 2.027 .020 & 5.273 .139 \\
& Exportaciones & 461.957 & 1.606 .249 & 1.223 .481 \\
\hline
\end{tabular}

Fuente: ALADI. Elaboración propia.

13.944 millones de dólares estadounidenses, o $14.8 \%$ del total invertido en el exterior" (Dirección General de Relaciones Económicas Internacionales de Chile [Direcon], 2014, p. 1). El establecimiento de la Alianza del Pacífico en el año 2011, ${ }^{8}$ ha constituido un nuevo escenario que estimula el fortalecimiento de las relaciones comerciales entre los dos países de cara a la región Asia Pacífico.

En el caso de Bolivia, las relaciones comerciales están enmarcadas por el Acuerdo de Complementariedad Económica entre Bolivia y Chile (ACE No. 22), el cual entró en vigencia en 1993. El ACE establece preferencias arancelarias y liberación de gravámenes. Si bien Chile es un importante destino para las exportaciones bolivianas y ocupa un lugar destacado en las importaciones, la importancia no es recíproca, de manera que para Chile, su vecino ocupa un lugar marginal como socio comercial.

Finalmente, hay una importante dinámica comercial entre Chile y Argentina. Según cifras de la Asociación Latinoamericana de Integración de 2012 (ALADI, 2013), para las exportaciones argentinas Chile representa el segundo destino en importancia, con $6.26 \%$ del total de las exportaciones; no así en importaciones, donde Chile sólo representa $1.48 \%$ del total. Entre tanto, Argentina constituye el destino del 1.59\% de las exportaciones chilenas y el tercer país de origen de las importaciones con $7.46 \%$ del total.

Por otra parte, la minería es un renglón clave de la economía regional, pero también del país, representando 12\% del PIB chileno en el año 2012

${ }^{8}$ La Alianza del Pacífico es una iniciativa de libre comercio y cooperación entre Chile, Perú, Colombia y México. El tratado contempla la eliminación de los aranceles en $92 \%$ de los productos a partir de su ratificación, mientras que para el $8 \%$ restante, que corresponde a productos agrícolas, se estableció un proceso gradual de eliminación arancelaria. La alianza contempla un mercado de al menos 210 millones de personas. 
(Banco Central de Chile, 2013). La configuración de una economía de enclave en el territorio norte del país mediante la explotación del salitre llevó a su incipiente inserción en la economía mundial durante el siglo XIx. Hasta hoy, la minería constituye el motor de su economía, especialmente en las regiones de Tarapacá y Antofagasta —extracción de cobre, cloruro de sodio y azufre- . Para Arica y Parinacota la industria manufacturera y el comercio, hoteles y restaurantes son las dos actividades económicas que mayor peso tienen en la economía regional. En general, la economía de esta zona se sustenta en recursos naturales y está poco diversificada (ver figura 3). La industria está principalmente ligada al recurso pesquero. La zona franca de Iquique, en la región de Tarapacá, y la condición de puerto libre de la ciudad de Arica, han generado dinámicas de comercio e intercambio de bienes que son vitales para su economía.

\section{Infraestructura y conectividad: La frontera norte e IIRSA}

La importancia de la integración económico-comercial de la Iniciativa para la Infraestructura Regional Suramericana (IIRSA) dentro de la estrategia de inserción internacional chilena está vinculada con el desarrollo de la infraestructura y la conectividad, la segunda variable que se ha propuesto como relevante en la relación del Estado chileno con la frontera norte. En el marco de la IIRSA, el territorio del norte de Chile es estratégico, tanto por integrar diversos ejes de conectividad como por permitir el paso hacia otros ejes de enlace con el resto de la región.

La IIRSA es un mecanismo de coordinación entre los países de la región para el desarrollo e integración de la infraestructura física transfronteriza, con el objetivo de ofrecer un mayor mercado a las empresas, aumentar el comercio intrarregional y mejorar la competitividad de Sudamérica con el resto del mundo. La precariedad de la infraestructura física es considerada una de las mayores problemáticas para la integración regional y el desarrollo económico; se considera que un aumento de 10 puntos porcentuales en los costos de transporte tiende a reducir los volúmenes de comercio en $20 \%$, y que los costos de transporte superan los costos arancelarios en Sudamérica (Comisión Económica para América Latina y el Caribe [CEPAL], 2013). 


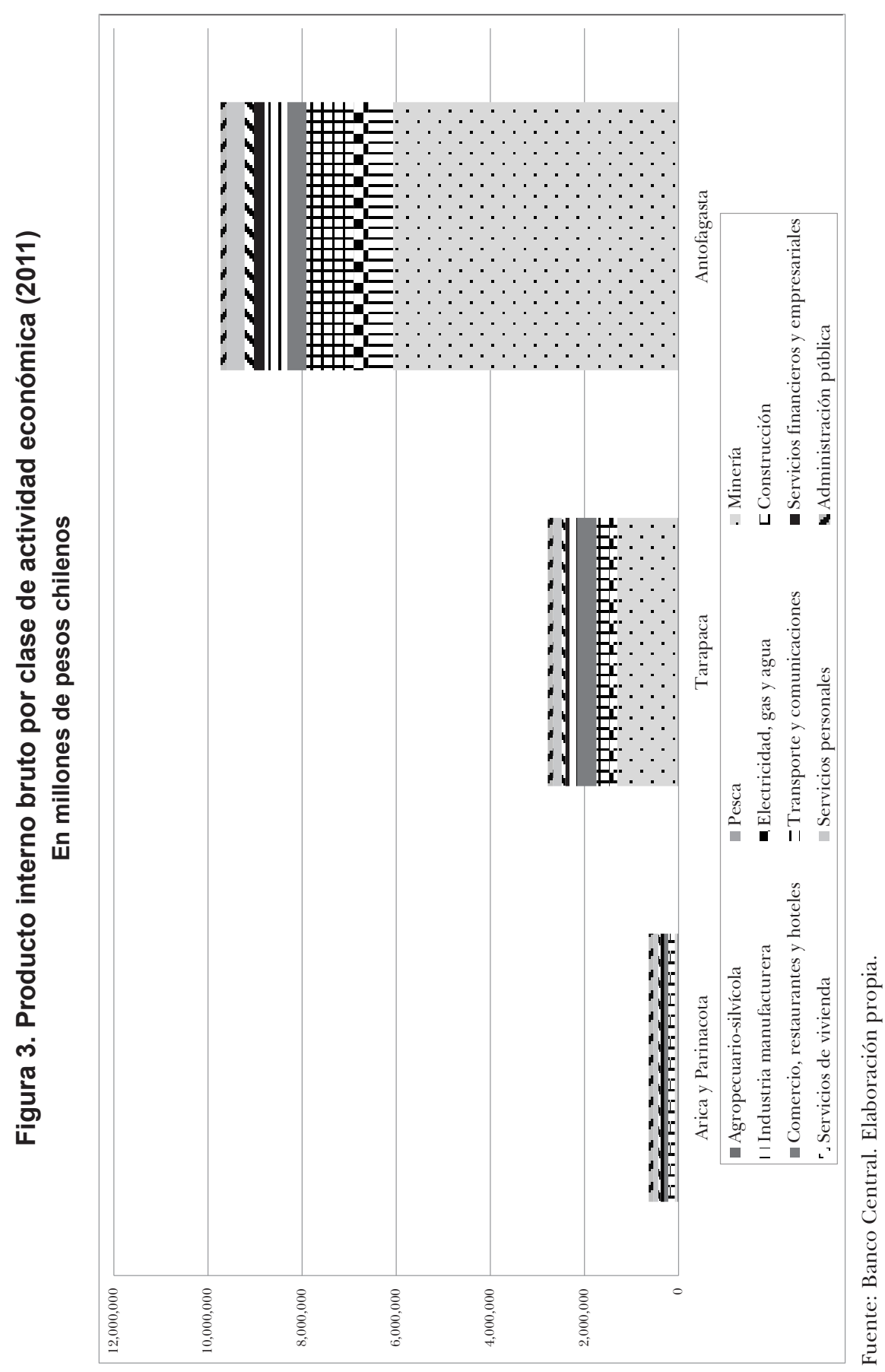


La planificación planteada por IIRSA está estructurada a partir de ejes de integración y desarrollo, entendidos como "franjas multinacionales de territorio en donde se concentran espacios naturales, asentamientos humanos, zonas productivas y flujos comerciales" (IIRSA, s.f., p. 1). Chile hace parte de los ejes: Mercosur-Chile, Interoceánico Central, Capricornio y del Sur (cuadro 2).

Tres de los ejes de integración donde Chile hace parte comprenden territorios del área fronteriza del norte país, configurándola como un importante corredor de bienes, servicios y personas. No obstante, esta dinámica de intercambio transfronterizo no es nueva en esta área, dado que en su condición de espacio de frontera históricamente ha sido lugar de migración, encuentro e intercambio constante. Lo que ha cambiado es el ritmo de esos flujos e intercambios, así como su lugar en el proyecto económico, tanto de Chile como de la región Suramericana. Dentro de la Agenda de Proyectos Prioritarios de Integración (API), ${ }^{9}$ dos involucran

\section{Cuadro 2. Ejes de IIRSA que incluyen a Chile}

\begin{tabular}{|c|c|}
\hline Eje & Composición \\
\hline Mercosur-Chile & $\begin{array}{l}\text { Vincula los principales centros económicos y los principales } \\
\text { puertos de ese territorio entre Chile, Argentina, Paraguay, } \\
\text { Uruguay y Brasil. }\end{array}$ \\
\hline $\begin{array}{c}\text { Eje Interoceánico } \\
\text { Central }\end{array}$ & $\begin{array}{l}\text { Atraviesa Suramérica conectando el Océano Atlántico con el } \\
\text { Pacífico a través de nodos en cinco países: Perú, las Regiones } \\
\text { xv, I y la Provincia Loa de la II Región de Chile, Bolivia, } \\
\text { Paraguay y Brasil. }\end{array}$ \\
\hline Eje de Capricornio & $\begin{array}{l}\text { Conformado por cuatro regiones y comprende territorio de } \\
\text { Brasil, Argentina, Paraguay, Bolivia y las regiones chilenas } \\
\text { de Tarapacá, Antofagasta y Atacama. }\end{array}$ \\
\hline Eje del Sur & $\begin{array}{l}\text { Integrado por Chile y Argentina, en este caso el eje abarca las } \\
\text { regiones VIII, Ix, XIV y x de Chile. }\end{array}$ \\
\hline
\end{tabular}

Fuente: Elaboración propia con datos de IIRSA (s.f.).

${ }^{9}$ La Agenda de Proyectos Prioritarios de Integración (API) consiste en un conjunto de 31 proyectos estructurados por un monto de inversión estimado en 16.713,8 millones de dólares estadunidenses, de carácter estratégico y de alto impacto para la integración física y el desarrollo socio-económico regional. Su objetivo es promover la conectividad de la región a partir de la construcción y operación eficiente de la 
a Chile: Corredor ferroviario bioceánico Paranaguá-Antofagasta y túnel binacional Agua Negra. El primero, ubicado en área de la frontera norte, es parte del Eje de Capricornio: "Tiene por objeto proporcionar una conexión ferroviaria multilateral para el transporte de cargas en el Eje de Capricornio entre los territorios considerados, en una traza que va desde el puerto de Antofagasta, en Chile, pasando por el norte argentino y por Paraguay, siguiendo por el territorio brasileño hasta el puerto de Paranaguá” (IIRSA, 2012, p. 66). A través del corredor se reducirán los costos de logística en el transporte y se fomentarán los intercambios comerciales. Nueve proyectos individuales conforman este API, el tramo de Chile corresponde a Antofagasta-Socompa (ver figura 4).

\section{La agenda de seguridad en el norte de Chile}

El último aspecto que se considera en este análisis es la seguridad. Al respecto, la Declaración de Seguridad de las Américas, aprobada en 2003 (Organización de los Estados Americanos [OEA], 2013), establece una visión de seguridad multidimensional para el hemisferio que abarca las amenazas convencionales y las nuevas amenazas. En ella se señala la necesidad de ampliar los enfoques tradicionales, dada la naturaleza diversa de las actuales amenazas a la seguridad que incluye aspectos políticos, económicos, sociales, de salud y ambientales, así como una ampliación en los objetos de referencia de la seguridad, donde no sólo se considera al Estado, sino al individuo y a las comunidades. Las amenazas, según la Declaración sobre la seguridad en las Américas (oEA, 2013).

- Amenazas tradicionales: Conflictos fronterizos, guerras interestatales, etcétera.

- Nuevas amenazas: El terrorismo, la delincuencia organizada transnacional, el problema mundial de las drogas, la corrupción, el lavado de activos, el tráfico ilícito de armas y las conexiones entre ellos; la pobreza extrema y la exclusión social de amplios sectores de la

infraestructura, atendiendo a criterios de desarrollo social y económico sustentable, preservando el ambiente y equilibrio de los ecosistemas (IIRSA, 2012, p. 5). 
Figura 4. Corredor ferroviario bioceánico Paranaguá-Antofagasta

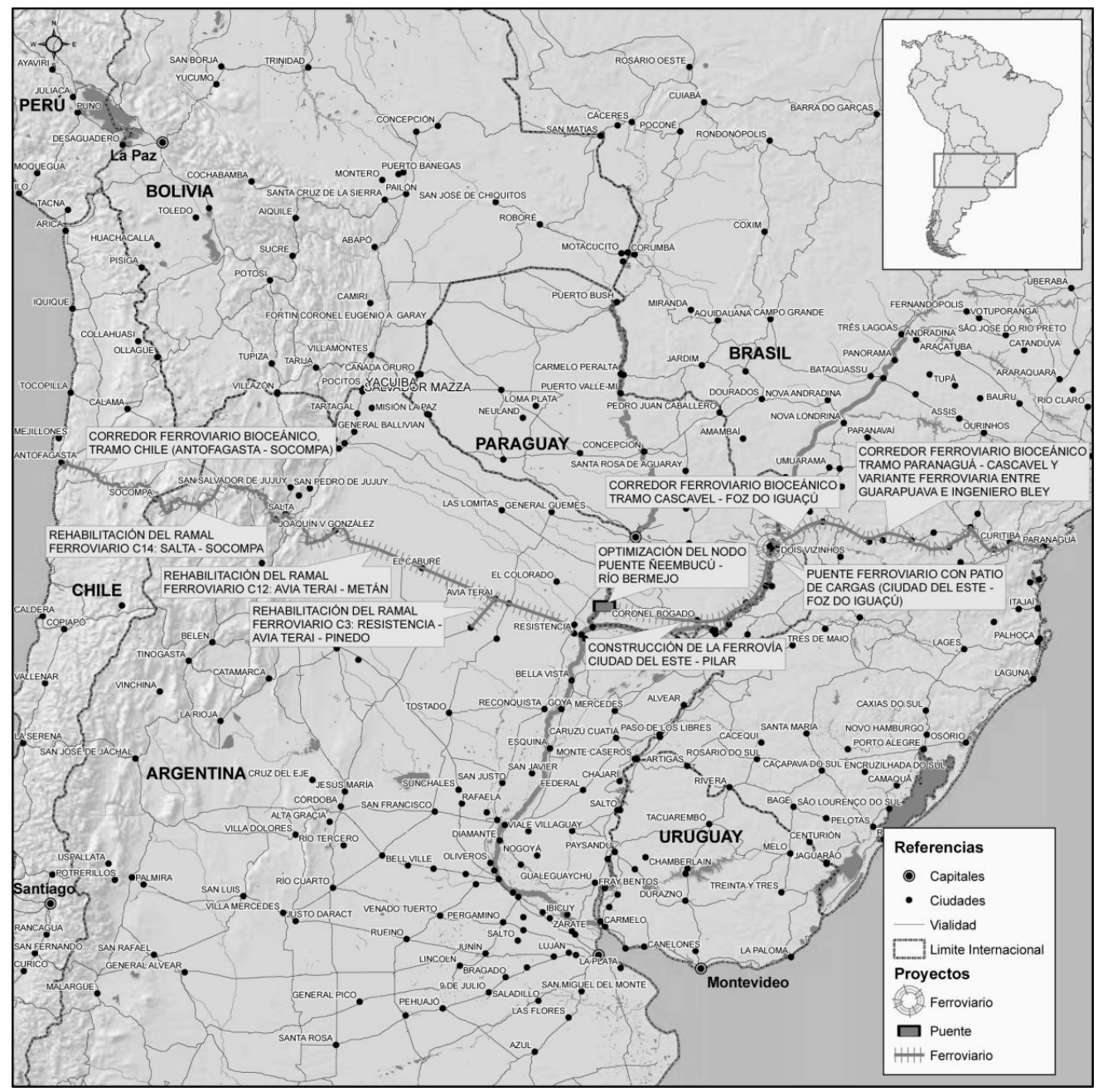

Fuente: IIRSA, 2014. 
población; el VIH/SIDA y otras enfermedades; el deterioro del medio ambiente; la trata de personas; los ataques a la seguridad cibernética; la posibilidad de que surja un daño en el caso de un accidente o incidente durante el transporte marítimo de materiales potencialmente peligrosos, incluidos el petróleo, material radiactivo y desechos tóxicos; y la posibilidad del acceso, posesión y uso de armas de destrucción en masa y sus medios vectores por terroristas.

Un amplio componente de lo que es definido como "nuevas amenazas" a la seguridad hace referencia a los actores y flujos transnacionales ilegales. "Los flujos ilícitos transnacionales de bienes, información y personas —el lado clandestino de la globalización- se han convertido en una fuente creciente de tensión y conflicto en la política mundial" (Andreas, 2004, p. 642), siendo considerados como una amenaza para la seguridad internacional y un desafío para el control y la aplicación de la ley por parte de las autoridades. A decir de Andreas (2004):

Como la economía global se ha convertido en más integrada e interdependiente, los estados han construido barreras contra el intercambio "indeseable" a través de las fronteras. Esto se ha hecho particularmente evidente en los esfuerzos para controlar la migración ilegal, las drogas prohibidas y las finanzas ilícitas (p. 643).

La frontera norte de Chile condensa las tensiones y dinámicas entre la agenda tradicional y la nueva agenda de seguridad. Así, en el año 2011, mientras el gobierno chileno anunció el establecimiento de una estrategia de seguridad fronteriza denominada "Plan Frontera Norte", con el objetivo de combatir el narcotráfico, contrabando y otros flujos ilícitos en las regiones de Arica y Parinacota, Tarapacá y Antofagasta, al norte del país (Cámara de Diputados de la República de Chile, 2013), el gobierno boliviano creó la Dirección de Reivindicación Marítima con el objetivo de llevar ante los tribunales internacionales el reclamo por una salida soberana al Océano Pacífico, plan que se concretó en el año 2013, cuando Bolivia presentó formalmente una demanda contra Chile ante la Corte Internacional de Justicia (CIJ). Esta demanda se sumó a la que desde el año 2008 había sido interpuesta por Perú en contra de Chile respecto del límite marítimo 
entre los dos países y sobre la que la CIJ profirió su fallo en enero de 2014. El 29 de abril de 2013, la CIJ comunicó la aceptación de la demanda de Bolivia por una salida soberana al Océano Pacífico contra Chile. No obstante, en julio de 2014, la demanda fue suspendida tras la presentación, por parte del gobierno chileno, de un recurso de excepción preliminar a la jurisdicción de la Corte respecto de su competencia. ${ }^{10}$

No se profundizará en los detalles de los diferendos territoriales y marítimos, bastará con señalar que esta clase de disputas están estrechamente ligadas con temas tales como el nacionalismo y la soberanía, alimentando hipótesis de desconfianza, conflicto y militarización. Empero, como bien señala Sotomayor (2009), en la región es poco probable un conflicto bélico por causa de estas problemáticas y los Estados cuentan con diferentes métodos e instancias para resolverlas de una manera pacífica, particularmente a partir de la década de 1990, los países de la región han optado más por recurrir a la cIJ.

Las problemáticas propias de la agenda tradicional de seguridad, se entrecruzan con el posicionamiento de una agenda caracterizada por las nuevas amenazas, entre ellas, el narcotráfico y el contrabando. El Plan Frontera Norte (PFN) representa de manera concreta la implementación de acciones por parte del Estado chileno con base en esa nueva agenda de seguridad. El plan fue anunciado por el gobierno en octubre de 2011 y en su primera fase comprende las regiones de Arica-Parinacota, Tarapacá y Antofagasta.

El PFN tiene como principal objetivo evitar el ingreso y la salida de droga y contrabando del país a través de puertos, sectores costeros no habilitados, complejos fronterizos y pasos no habilitados. Para ello, está estructurado a partir de los siguientes ejes: fortalecimiento de los complejos fronterizos, control de los pasos no habilitados, fortalecimiento de la presencia en la frontera marítima, cooperación con los países vecinos contra el crimen organizado y la mejora de la capacidad operativa de Carabineros y la Policía de Investigaciones. La perspectiva del plan se basa en una consideración de la seguridad a partir de las amenazas irregulares o nuevas, donde se busca evitar el paso de determinados bienes y personas a través de las fronteras.

${ }^{10}$ A la excepción de competencia de la cIJ, solicitada por Chile, falta la presentación del descargo de la parte boliviana que debe presentarse el 14 de noviembre de 2014. 
La dimensión geográfica y el contexto regional son una de las razones de mayor peso para el desarrollo del PFN. En este sentido, al exponer la importancia del plan frente a una comisión de diputados, el subsecretario del Interior, Rodrigo Ubilla, manifestaba:

En el norte de Chile 947 kilómetros corresponden a la frontera con dos países (Perú y Bolivia) que producen el $54 \%$ de la cocaína en el mundo; mientras que 400 kilómetros de la frontera con Argentina, en la región de Antofagasta, tienen conexión con Brasil y Paraguay, éste último uno de los mayores productores de marihuana prensada (Cámara de Diputados de la República de Chile, 2013, p. 14).

Según cifras de la Organización de las Naciones Unidas (ONU) para el año 2012, Perú es el mayor productor de hoja de coca con 60400 hectáreas, seguido por Colombia con 48 000. Entre tanto, en el año 2011, Bolivia tenía 27200 hectáreas de coca (Oficina de las Naciones Unidas contra las Drogas y el Delito [ONudD], 2013). A las consideraciones sobre el contexto regional, se suma la dinámica del tráfico de drogas ilícitas en el territorio chileno; al respecto las cifras evidencian que Chile se ha convertido en una zona de tránsito de drogas ilícitas así como de consumo (Ministerio del Interior y Seguridad Pública, 2013).

Centrándose en las cifras respecto del tráfico, un balance sobre los decomisos efectuados entre 2009 y 2012 evidencia un incremento en los de pasta base de cocaína, la marihuana procesada y las plantas de marihuana decomisadas. Este aumento puede explicarse por dos motivos: el mayor control por parte de los aparatos policiales y/o el incremento en los flujos de drogas ilícitas en el país (cuadro 3).

Desde una perspectiva regional, los decomisos de clorhidrato de cocaína se concentran en las regiones Metropolitana, Arica y Parinacota y Tarapacá. En segundo lugar, las regiones con mayor decomiso de pasta base de cocaína son la Región Metropolitana, Tarapacá y Antofagasta. Antofagasta también es una de las regiones con mayor decomiso de marihuana procesada. Las regiones de Arica y Parinacota y Tarapacá también son algunas de las que presentan mayores decomisos de fármacos.

En este contexto, a través del PFN se busca evitar el ingreso de drogas ilícitas al territorio chileno, mediante una mayor coordinación 
Cuadro 3. Decomisos según tipo de drogas, 2009-2013

\begin{tabular}{|cccccc|}
\hline Tipo de droga & 2009 & 2010 & 2011 & 2012 & 2013 \\
\hline C. de cocaína (kilos) & $2.654,89$ & $2.808,01$ & $2.392,14$ & $3.150,18$ & $2.932,77$ \\
Pasta base de Cocaína & $5.695,79$ & $7.127,07$ & $7.059,77$ & $9.675,41$ & $10.798,40$ \\
$\begin{array}{c}\text { Marihuana procesada } \\
\text { (kilos) }\end{array}$ & $13.928,46$ & $8.144,62$ & $145.65,59$ & $14.550,82$ & $23.305,53$ \\
$\begin{array}{c}\text { Plantas de marihuana } \\
\text { (unidades) }\end{array}$ & 196.412 & 172.618 & 26.6015 & 23.5428 & 28.8379 \\
Fármacos (unidades) & 84.619 & 393.064 & 167.885 & 116.361 & 68.741 \\
Heroína (kilos) & 0,1 & 0,0 & 0,0 & 0,0 & 0,0 \\
\hline
\end{tabular}

Fuente: Ministerio del Interior y Seguridad Pública (2014).

interinstitucional, el uso de tecnología y la cooperación con los países vecinos. En total, el Plan Frontera Norte supone una inversión entre 2011 y 2014 de 35000 millones de pesos chilenos, para fortalecer las fronteras terrestres y marítimas de las tres regiones involucradas. A comienzos de 2013, el gobierno chileno anunció la continuidad del plan y la inclusión de la región de Atacama en éste.

La naturaleza interméstica de problemáticas como el narcotráfico y la necesidad de emprender medidas conjuntas ha resultado en una situación paradójica en la que, por un lado, la nueva agenda de seguridad genera incentivos para el acercamiento y la cooperación, a pesar de las disputas territoriales latentes, mientras que, por otra parte, debido a la visión negativa y de desconfianza frente al "otro" que caracteriza la relación de Perú y Bolivia con Chile, y a las asimetrías en términos de políticas y de capacidad institucional, ${ }^{11}$ ha traído consigo nuevos espacios de desencuentro entre los tres países.

${ }^{11}$ Las asimetrías, ya sean de tipo económico o institucional, afectan las relaciones entre los estados y las políticas frente a las amenazas trasnacionales. En ciertos casos éstas generan incentivos para que las actividades ilícitas sean toleradas, bien sea por actores estatales nacionales o locales. En el ámbito institucional, las diferencias entre la capacidad estatal para la implementación de políticas de seguridad generan conflictos entre los Estados. 


\section{Conclusiones}

Las dinámicas propias de la globalización y la integración regional cuestionan la idea de las fronteras como límites territoriales y de soberanías estatales de carácter rígido; las fronteras son cada vez más porosas, constituyendo un espacio donde convergen diversos actores, fenómenos y procesos sociales. Sin embargo, esto no supone la desaparición de las fronteras o la retirada del control estatal en dichos territorios. Por el contrario, la administración de las fronteras sigue siendo una de las tareas del Estado, la dimensión territorial mantiene su importancia y lo que se advierte es un cambio en las funciones del control estatal y las formas de territorialización. En este sentido, la revisión de la literatura sobre el tema señala lo que Aguiar (2010) denomina "neoliberalismo selectivo", en el que se promueve la anulación de límites para los flujos de bienes, servicios y capital financiero, a la vez que se construyen nuevas barreras para ciertas personas y bienes considerados ilegales.

El territorio correspondiente a la frontera norte de Chile constituye a la vez una frontera interna e internacional. En su condición de frontera interna, históricamente se ha caracterizado por su aislamiento y marginalidad, "zona extrema" y "localidad aislada" son los calificativos que se han usado desde la institucionalidad estatal para designarla. Desde mitad del siglo xx, el Estado chileno ha puesto en marcha políticas para incentivar el desarrollo e integración de esta zona, las cuales no han logrado su cometido con mayor éxito. Sin embargo, como frontera internacional, las dinámicas económicas y sociales en el marco de la globalización han generado una inusitada importancia de esta zona como pivote para el desarrollo y la integración en torno a tres variables: comercio y exportación de bienes primarios, infraestructura y seguridad.

En torno a estas variables se ha dado también un cambio en las formas de territorialización del Estado chileno. El territorio del norte es de importancia estratégica debido a sus recursos minerales, así como por su posición geográfica donde constituye un corredor que conecta a Chile con el resto de la región Suramericana, de manera que desde la perspectiva económica y comercial es una zona clave. No obstante, la aceleración en los flujos e intercambios de la economía legal, ha ido acompañada también por el posicionamiento de nuevos fenómenos considerados problemáticos 
desde una perspectiva de seguridad; es así como la política de seguridad del Estado chileno ha dejado de enfocarse en las amenazas tradicionales (disputas territoriales) para considerar temas como el narcotráfico o la inmigración no autorizada, lo que ha traído consigo un cambio en la forma en que el Estado ejerce sus funciones de control y represión.

\section{Bibliografía}

Aguiar, J. C. (2010). Stretching the border: Smuggling practices and the control of illegality in South America (New Voices Series 6). Chile: Global Consortium on Security Transformation.

Andreas, P. (2003). Redrawing the line. Borders and security in the Twenty-first Century. International Security, 28(2), 78-111.

Andreas, P. (2004). Illicit international political economy: The clandestine side of globalization. Review of International Political Economy, 11(3), 641-652.

Aranda, G., Ovando, C. y Corder, A. (2010). Experiencias paradiplomáticas en la región de Tarapacá y su proyección subregional. Estudios Internacionales, 43(165), 33-73.

Asociación Latinoamericana de Integración (ALADI) (2013). Sistema de Información de Comercio Exterior (SICOEX). Recuperado de: <http://consultawebv2.aladi.org/ sicoexV2/jsf/home.seam>

Bartolomé, M. (2005). Antropología de las fronteras en América Latina. Ameriquest, 2(1), 1-17.

Bello, D. (2012). Alianza Estratégica Aymaras sin Fronteras: Una respuesta territorial a los desafíos de la glocalización. Tinkazos, 15(32), 147-164.

Biblioteca del Congreso Nacional de Chile (BCN) (2007). Historia de la Ley № 20.175 Crea la XV Región de Arica y Parinacota y la provincia del Tamarugal en la Región de Tarapacá. Recuperado de: <http://www.leychile.cl/Navegar/scripts/obtienearc hivo?id = recursoslegales/10221.3/543/1/HL20175.pdf >

Biblioteca del Congreso Nacional de Chile (BCN) (2011). Politica nacional para el desarrollo de localidades aisladas. Recuperado de: < http://transparencia.bcn. cl/obtienearchivo?id=repositorio/10221/12189/1/91462_Minuta_PoliticaNacional-para-el-desarrollo-de.doc $>$

Cámara de Diputados de la República de Chile (2013). Informe de la Comisión Especial Investigadora acerca de la implementación del denominado "Plan Frontera Norte". Santiago: Biblioteca del Congreso Nacional de Chile.

Cano, V. y Soffia, M. (2009). Los estudios sobre migración internacional en Chile: Apuntes y comentarios para una agenda de investigación actualizada. Papeles de Población, 15(61), 129-167. 
Correa, L. y García, V. (2012). Aunque las aguas nos dividan: Las relaciones chilenobolivianas y la construcción de una agenda común. Revista Latinoamérica, (54), 75-110.

Comisión Económica para América Latina y el Caribe (CEPAL) (2013). Comercio internacional y desarrollo inclusivo. Construyendo sinergias. Recuperado de: $<$ http://www.cepal.org/publicaciones/xml/4/49724/ComercioInternacionalyD esarrolloInclusivo.pdf>

Comisión Nacional sobre Fronteras Interiores al Desarrollo Nacional (1995). Proposiciones de la Comisión Nacional sobre Fronteras Interiores al Desarrollo Nacional a S.E. el Presidente de la República Dn. Eduardo Frei Ruiz-Tagle. Santiago: Autor.

Comité de Inversiones Extranjeras de Chile (2012). Estadísticas. Recuperado de: $<$ http://www.ciechile.gob.cl/es/inversion-en-chile/estadisticas/>

Dirección General de Relaciones Económicas Internacionales de Chile (Direcon) (2014). Presencia de Inversión Directa de Capitales Chilenos en Perú 1990-diciembre 2013. Recuperado de: < http://www.direcon.gob.cl/wp-content/ uploads/2014/10/004_Presencia-ID-Cl-en-Per\%C3\%BA-1990-dic2013.pdf>

Fuentes, C. (2008). Fronteras calientes. Foreign Affairs Latinoamérica, 8(3), 12-21.

Fuentes, C. (2011). Balance crítico de la política exterior de Chile. En J. Ensignia, C. Fuentes y M. Fernández (Eds.), Política Exterior en el Chile post-concertación. ¿Quo vadis? Santiago: FESCOL.

Garay, C. (2004). Estados débiles y espacios vacíos. El caso chileno. Security and Defense Studies Review, 4(2), 91-113.

González, S., Rouviere, L. y Ovando, C. (2008). De "Aymaras en la frontera" a "Aymaras sin fronteras". Los gobiernos locales de la triple frontera andina (Perú, Bolivia y Chile) y la globalización. Diálogo Andino, 31, 31-46.

González, S. y Ovando, C. (2008). Hacia un nuevo pensamiento integracionista latinoamericano: Aproximación a una lectura de segundo orden. Polis, 7(21), 265-285.

González, S. (2009). El Norte Grande de Chile y sus dos Triple-Fronteras: Andina (Perú, Bolivia y Chile) y Circumpuneña (Bolivia, Argentina y Chile). Cuadernos Interculturales, 7(13), 27-42.

Grimson, A. (2000). Pensar las fronteras desde las fronteras. Nueva Sociedad, (170), 162-167.

Grimson, A. (2003). La nación después del (de)constructivismo. Nueva Sociedad, $184,33-45$.

Grimson, A. (2004). Fronteras, naciones y región. Ponencia, Foro Social de las Américas, Quito, julio.

Harvey, D. (1990). Between space and time: Reflections in the geographical imagination. Annals of the Association of American Geographers, 80(3), 418-434. 
Iniciativa para la Integración Regional Suramericana (IIRSA) (sin año). Ejes de integración y desarrollo. Recuperado de: <http://www.iirsa.org/Page/Detail?menu ItemId $=58>$

Iniciativa para la Integración Regional Suramericana (IIRSA). (2012). Agenda de Proyectos Prioritarios de Integración. Estado de Avance. III Reunión Ordinaria del Cosiplan. Recuperado del sitio de Internet de: < http://www.iirsa.org/ admin_iirsa_web//ploads/Documents/rc.lima12_api_informe_avance.pdf >

Instituto Nacional de Estadísticas (INE) (2012). Resultados Preliminares Censo de Población y Vivienda 2012. Santiago: Autor.

Machado, L. (2000). Limites e fronteiras: Da alta diplomacia aos circuitos da ilegalidade. Revista Territorio, 8, 9-29.

Machado, L., Reyes, A. y Rego, L. (2009). Building walls, breaking barriers: Territory, integration and the rule o law in frontier zones. Journal of Borderlands Studies, 24(3), 97-114.

Martínez, A. (2014). Fronteras interiores: Una realidad ligada a la seguridad y defensa. Recuperado de: <http://www.anepe.cl/2012/03/fronteras-interiores-una-realidadligada-a-la-seguridad-y-defensa/>

Ministerio de Defensa de Chile (2010). Libro Blanco de la Defensa. Santiago: Autor. Ministerio del Interior y Seguridad Pública (2013). Informe nacional. Procedimientos policiales por infracción a la Ley de Drogas Núm. 20000. Santiago: Autor.

Ministerio del Interior y Seguridad Pública. (2014). Informe nacional. Procedimientos policiales por infracción a la Ley de Drogas Núm. 20000. Santiago: Autor.

Navarrete, B. e Higueras, V. (2014). Chile desde la teoría secuencial de la descentralización, 1990-2010. Convergencia. Revista de Ciencias Sociales, 21(66), 179-202.

Newman, D. (2003). On borders and power: a theoretical framework. Borderlands Studies, 18(1), 13-25.

Newman, D. (2006). The lines that continue to separate us: Borders in our "borderless" world. Progress in Human Geography, 30(2), 143-161.

Quezada, A. (2010). Inserción internacional de Chile en la post-Guerra Fría. Revista Enfoques, VIII(12), 119-134.

Ramírez, S. y Cepik, M. (Eds.) (2004). Agenda de Seguridad Andino Brasileña: Primeras aproximaciones. Bogotá: FES, IEPRI, Universidad Federal Rio Grande do Sul.

Oficina de las Naciones Unidas contra las Drogas y el Delito (ONudD) (2013). Informe Mundial de Drogas. Recuperado de: <http://www.unodc.org/doc/ wdr2013/World_Drug_Report_2013_Spanish.pdf>

Organización de los Estados Americanos (OEA) (2013). Declaración sobre Seguridad en las Américas. Recuperado de: < https://www.oas.org/es/ssm/CE00339S03. $\operatorname{pdf}>$ 
Ovando, C. y Álvarez, G. (2011). La dimensión fronteriza de la política exterior de Chile: Inmovilidad y emergencia de nuevas dinámicas. Estudios Fronterizos, 12(24), 75-102.

Szary, A. (1997). Regiones ganadoras y regiones perdedoras en el retorno de la democracia en Chile: Poderes locales y desequilibrios territoriales. Revista EURE, 23(70), 59-78.

Tapia, M. (2012). Frontera y migración en el norte a partir del análisis de los censos población. Siglos xix- Xxi. Revista de Geografía Norte Grande, (53), 177-198.

Sotomayor, A. (2009). Forum shopping and territorial dispute settlement in Latin America. Ponencia, xxi Congreso de la Asociación Mundial de Ciencia Política, Santiago, junio.

Villafuerte, D. (2009). La centralidad de las fronteras en tiempos de mundialización. Comercio Exterior, 59(9), 693-703.

Wilson, T. y Donnan, H. (1998). Border identities: Nation and State at international frontiers. Cambridge: Cambridge University Press. 